\title{
Possibilities of physical medicine in the treatment of wound of tarsal joint
}

\author{
Jaroslaw Pasek ${ }^{1,2}$, Aleksander Sieron ${ }^{2}$ \\ 'Academy of Jan Dlugosz in Czestochowa, Institute of Physical Education Tourism and Physiotherapy, Czestochowa, Poland \\ ${ }^{2}$ School of Medicine with the Division of Dentistry in Zabrze University of Silesia in Katowice Chair and Department \\ of Internal Diseases Angiology and Physical Medicine, Bytom, Poland
}

\begin{abstract}
The treatment of wounds that are hard to heal still poses a serious interdisciplinary medical and sociological problem. Intense development of methods belonging to physical medicine has been noted recently. The paper presents beneficial results of treatment of a 69-year-old female patient with persisting chronic wound in the right lower limb, as consequence of a surgery of tarsal joint (arthrodesis). In the treatment, variable magnetic fields and light therapy (ledtherapy) procedures were applied for 17 weeks, leading to complete healing of the surgical wound. These are treatment methods which in many cases allow a reduction of treatment time and positively influence life quality of patients undergoing treatment. The above applies to illnesses and injuries of locomotor system, diseases affecting soft tissues, as well as chronic wounds. The applied physiotherapeutic method contributed to complete healing of the lesion, abolishment of the ailments suffered, as well as improved life quality of the treated female patient.
\end{abstract}

Key words: chronic wounds, magnetic fields, light therapy, rehabilitation

Acta Angiol 20 I5; 21, 4: 132-135

\section{Introduction}

Undoubtedly, the ever frequent use of physical medicine methods in many fields of medicine is a fact. A wide spectrum of procedures enables instituting suitable treatment, reducing treatment time, as well as social and economic costs of treatment. The above applies to pathological states (injuries of the locomotor system, soft tissues, chronic wounds) [I, 2]. So far, medicine has not had any golden mean in the treatment of chronic wounds. In many cases such treatment requires patience and perseverance, from both patient and the treating physician [3]. In Poland, the problem of chronic wounds affects some 500 thousand people a year, those persons suffer for months, not only because of the pain and progressing disability, but also social dysfunction. Despite the substantial progress in discovering processes which are responsible for wound formation, the results of chronic wound treatment are still not completely satisfactory [4].

A consequence of every surgery is a wound which should heal by itself. Some wounds, however, do not heal properly (for various reasons). In addition, the continuous piercing and cumbersome post-surgery pain significantly deteriorate the patient's well-being and life quality. The disrupted tissues may experience independent vasospasms, slowing of blood flow. Also, oedemas and inflammations may develop, along with gathering of exudates, which are toxic for tissues. All that results in the wound not healing as it should, while the process of treatment gets prolonged. Local tissue defects or extensive necrosis are most often the causes of numerous complications, often life threatening, exerting substantial influence upon all the subsequent life of the patient [5].

Address for correspondence: Jaroslaw Pasek, School of Medicine with the Division of Dentistry in Zabrze University of Silesia in Katowice Chair and Department of Internal Diseases Angiology and Physical Medicine, Poland, Stefana Batorego I5, 4I-902 Bytom, tel.: +4832786 I6 30, e-mail: jarus_tomus@o2.pl 
In accordance to CEAP (Clinical Etiologic, Anatomic, Pathophysiologic), the treatment of chronic wound comprises surgical and local treatment, connected with pharmacotherapy, treatment by compression, and properly selected kinesiotherapy exercises. In the entire programming and treatment process, an indispensable element in line with the contemporary knowledge should be physical medicine procedures $[4,6]$.

For a few years now, clinical experiments have been carried out to determine the effectiveness of magnetic field application in wound treatment. That applies to physical therapy methods, whose action is not only effective, but also free from adverse effects [7, 8].

The beneficial profile of magnetic field activities in wound treatment stems from their biological effects, among which there are: intensification of oxygen utilisation and tissues respiration, intensification of anaerobic respiration in ischaemic tissues, vasodilative and angiogenetic activity, causing the development of collateral circulation in areas of wound formation. In addition, magnetic fields intensify the processes of regeneration and repair, stimulate the epidermisation process, as well as inhibit infections [9, 10].

Application of extremely low frequency variable magnetic field ELF-MF is applied, together with non-laser optical radiation (ledtherapy), by means of innovative magnetic and light applicators [ II, 12]. The mechanism behind the therapeutic influence in wound treatment consists of stimulating the regeneration of damaged blood vessels that serve the purpose of micro-circulation, improvement of local blood supply in tissues, as well as improvement of blood rheological properties, increase of collagen production in fibroblasts, and acceleration of mitosis in the cells of epidermis germinative layer (stimulation of epidermisation). Even during the first procedures, one could notice the intensification of wound cleaning by removal of necrotic tissues, inhibition of purulent discharge spots, which got gradually covered with fresh epidermis [II].

\section{Aim of the study}

The aim of the study was to present the beneficial results of treatment of a 69-year-old female patient with chronic wound in the right lower limb, as consequence of a surgery of tarsal joint (arthrodesis) to whom the procedures of variable magnetic fields and light therapy have been applied with good results.

\section{Case study}

The female patient, 69-year-old has been admitted to the School of Medicine with the Division of Dentistry

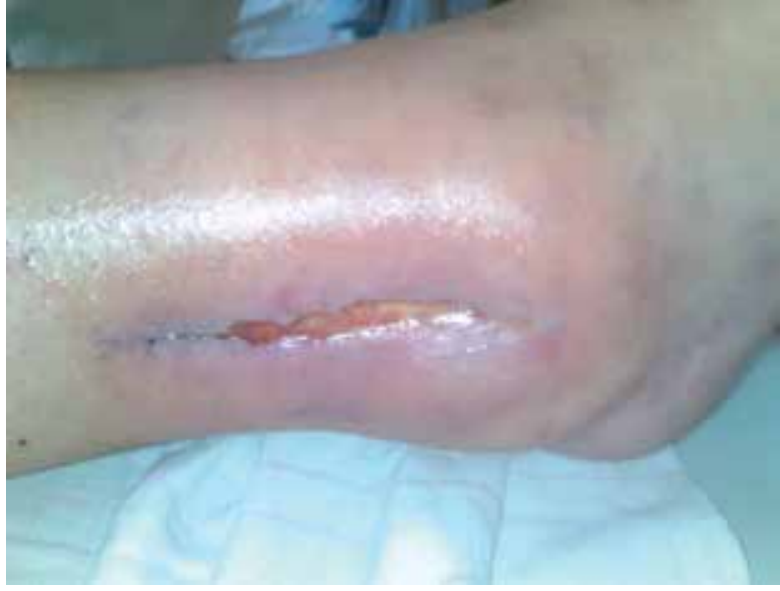

Figure I. The local wound state before the variable magnetic fields and light therapy procedures

in Zabrze, University of Silesia in Katowice, Chair and Department of Internal Diseases, Angiology and Physical Medicine due to a chronic wound in tarsal joint area, established several months earlier. Orthopaedic intervention at patients was in planned time executed. Until the time of admission, the patient had been treated with various effects (dermatology and surgery ward), yet her leg never healed completely. Before physical procedures hydrocolloid dressings were applied on the wound and broad spectrum antibiotic was administered. Due to difficulties in surgical wound healing, the patient was referred to our Clinic for further treatment.

On admission to the Clinic, besides the diagnostics (physical examination, consultation of a specialist in internal medicine, angiologist and vascular surgeon). At patients was generalization atherosclerosis of lower limbs and postphlebitis syndrome affirmed. The feature of insufficiency venous system was noted. Pulse on level foot perceptibility. At patients was $A B I$ - ankle brachial index - examination not executed. A cycle of variable magnetic fields and light therapy procedures was instituted. The consulting surgeon cleaned the wound in admission room conditions. Following that, physical treatment of the patient was initiated. During the preliminary examination, vast and trophic skin lesions, discharge of purulent secretion, as well as characteristic putrid smell of the lesion were noticed (Fig. I).

Before starting the therapy, the patient filled a concise pain intensity questionnaire, using the VAS scale ( 10 - most intense, I — least intense). The assessment concerned initial pain sensations experienced in the last three weeks. The result obtained has been assessed as very intense, according to the scale (10 points). 


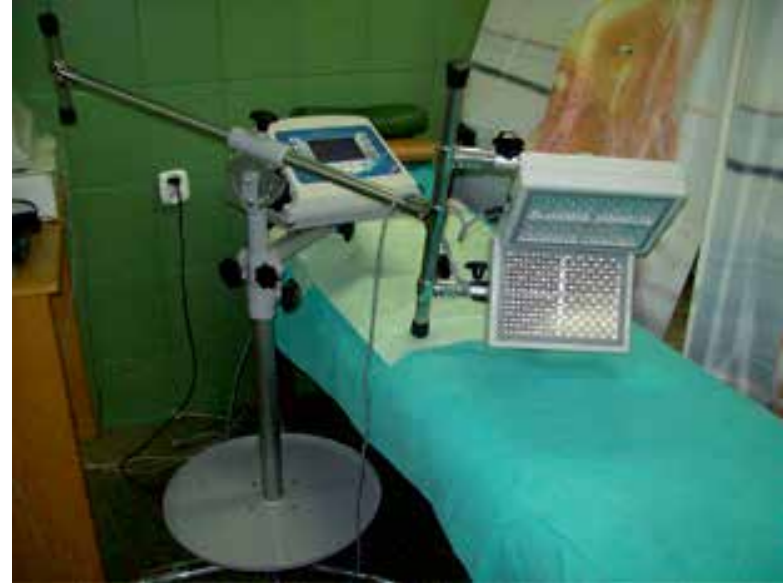

Figure 2. The Viofor JPS Standard device for variable magnetic fields and light therapy procedures, Med \& Life, Poland

\section{Physical treatment}

The patient was subjected to variable magnetic fields with light therapy procedures, using Viofor JPS Standard physical therapy device (Fig. 2) with IR panel applicators. The application was at the wound area, placing the applicators some $2-3 \mathrm{~cm}$ away from the surface to be exposed to radiation (the procedure was performed with the patient sitting). The procedures were performed in three series of 15 procedures each, with weekend breaks. The time interval between the two series of procedures was 4 weeks.

The luminous energy was produced by 280 specially selected high-energy LEDs, emitting light waves and energy that provide most beneficial therapeutic results. The device functioned in the Magnetic \& Light mode, emitting variable magnetic field with infrared radiation IR (wavelength $-850 \mathrm{~nm}$ ) for 12 minutes to the wound area, with the constant frequency of $181.8 \mathrm{~Hz}$ and settings of M2P2 controller, the magnetic field intensity amounted to 6 . The procedures were performed in outpatient mode, 2 times a day, daily for 3 weeks. The interval between series was 4 weeks. After each procedure the wound was dressed with a protective dressing. The patient was in recumbent position during the procedures. The physical treatment was combined with pharmacotherapy (anticoagulant, a drug improving blood flow and broad spectrum antibiotic).

M2 - application with increasing magnetic field intensity (magnetic field intensity increases every 10 or I 2 seconds, to a pre-selected level, in cycles during the application procedure).

P2 - JPS system with two types of impulses having the frequency of $180-195 \mathrm{~Hz}$.

\section{Results}

In the course of the first and second therapeutic session the patient reported significant alleviation of the pain experienced. Also, reduction of the purulent deposit could have been observed. The wound secretion changed into serous type, gradually the characteristic smell of decay disappeared. Also, fine-grain pink granulation appeared, which gradually began to cover the wound with new epidermis. Very weak currents, that formed during interaction with piezoelectric substances, occurred to stimulate the activity of cells, which in turn promoted vasodilatation (direct influence upon relaxation of smooth muscular coat of vessels, with substantial stimulation of neo-angiogenesis). Increased blood supply for tissues influenced their better oxygen supply positively, which evidently stimulated the processes of reconstruction and healing of structures.

After 5 months ( 17 weeks) the treatment was completed. The result obtained demonstrated full remission of the pain experienced, reduction of the inflammation process, as well as complete healing of the wound. In the course of the treatment, dynamic and positive healing of the lesion has been observed. The final result of the therapy performed is presented on Figure 3. In the course of treatment, the patient reported no side effects of the therapy she was undergoing. After the treatment was completed, significant improvement of clinical condition has been obtained in that female patient, as well as improvement of the patient's mental condition, as the wound has visibly healed.

\section{Discussion of results}

Recent years saw a notable progress in the field studies concerning the nature of processes taking place in the course of healing of acute and chronic wounds [2, 9, 13]. The five months of treatment led to complete healing of the chronic wound. Application of variable magnetic fields and light therapy procedures contributed to reduction of the pain experienced, complete healing of the wound, as well as improvement of the patient's life quality [14]. After the completion of treatment, the patient reported that she was able to move and apply load to the limb, which substantially simplified the patient's activities of daily living. From that moment on, significant improvement has also been obtained in the patient's clinical condition, as well as improvement of the psychical condition, in connection with the noted healing of the lesion.

The process of wound healing, including the wounds with various aetiologies, can be influenced by many delaying factors, due to metabolic deficiencies and attenuation of physiological reactions. Searching 


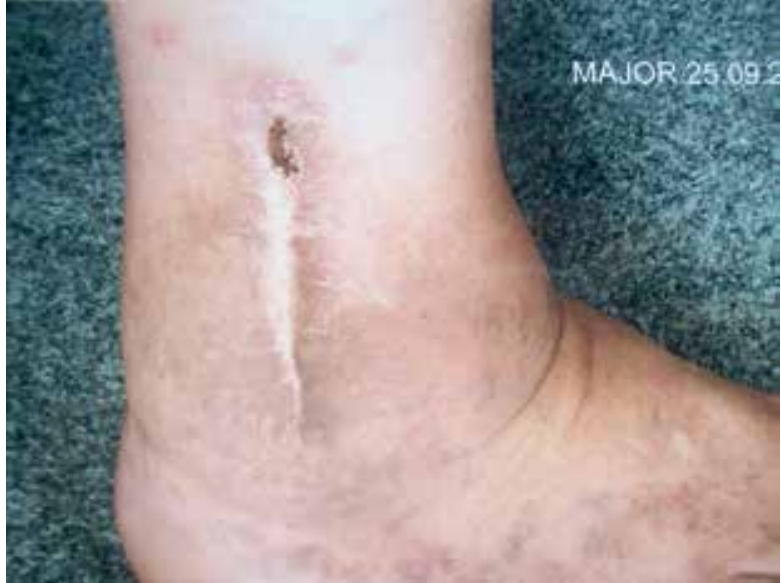

Figure 3. The local wound state after the end of variable magnetic fields and light therapy procedures

for various solutions that may give beneficial results of treatment is an ambitious clinical challenge, and requires profound knowledge and skills in treatment planning. Lack of patients' persistence with the therapy and failure to follow subsequent nursing care recommendations leads to quick recurrence of the disease. Thus patients should be informed that the physical procedures applied deal with the symptoms of the disease, not its causes [I, 3].

The mechanism of therapeutic action of magnetic fields and light therapy in such cases consists of stimulation the regeneration of destroyed network of vessels responsible for micro-circulation, and enhancing of local blood supply for tissues. Increased oxygen uptake is related to stimulation of tissue respiration processes, synthesis of DNA, and acceleration of mitotic cycle. Implemented at proper time, with parameters suitably selected, it is a useful treatment method in a complex treatment. With minimum contra-indications for its application, that kind of therapy may in numerous cases be the only effective type of treatment [II, I5].

Therapeutic results achieved through the application of this method may be highly satisfactory, as they are connected with fast activity (analgesic and anti-inflammatory effect), without physical contact and without pain, which is very important for the patient. The search for various solutions, which may bring about positive results of treatment, is a substantial clinical challenge, which in turn requires considerable knowledge and skills in planning of treatment $[8,16]$.

\section{Conclusions}

I. The application of variable magnetic fields and light therapy is an efficient physiotherapeutic method in the treatment of chronic wounds after surgery of tarsal joint.

2. Biological effects may help millions of suffering patients from high risk group; it may also generate enormous savings in health care. Even a short time of application of above procedures results in substantial overall clinical improvement, as well as local improvement, providing beneficial cosmetic results.

\section{References}

I. Vovden P, Apelqvist J, Moffat Ch (2008) Wound Complexity and Healing. In: Position Document of EWMA, Hard-to-heal wounds: a holistic approach, pp. 2-9.

2. Sieron A, Pasek J (20II) Magnetic fields in the treatment of wounds. Practical Rehabilitation; 4: 48-5I.

3. Schindzielorz M, Schmidt $M$ (20I I) Implementing new concepts in wound management. Interdisciplinary wound management. Pflege Z.; 64: 158-160.

4. Sieroń A, Cierpka L, Rybak Z, Stanek A (2009) Angiology textbook. Wydawnictwo Alfa-Medica Press, Bielsko-Biała.

5. Raffetto JD, Marston WA (20II) Venous ulcer: what is new? Plast Reconstr Surg; 127: 279-288.

6. Cutting K, White R, Hoekstra H (2009) Topical silver impregnated dressings and the importance of the dressing technology. Int Wound J; 6: 17-23.

7. Cieślar G, Nowak M, Kawecki M, Glinka M, Sieroń A (2005) Application of variable magnetic fields in wounds treatment. Leczenie Ran; 2: 99-106.

8. Sieron A (2002) The use of magnetic fields in medicine. Wydawnictwo Alfa-Medica Press, Bielsko-Biała.

9. Costin GE, Birlea SA, Norris DA (20I2) Trends in wound repair: cellular and molecular basis of regenerative therapy using electromagnetic fields. Curr Mol Med; 12: 14-26.

10. Sieroń A, Glinka M (1999) The influence of variable magnetic fields on wounds healing. Polish Balneology; I: 75-180.

II. Sieroń A, Pasek J, Mucha R (2007) Magnetic fields and light energy in medicine and rehabilitation — magnetoledtherapy. Polish Balneology; I: I-7.

12. Pasek J, Sieroń A (20II) Ledtherapy. Practical Rehabilitation and Physiotherapy; 13: 52-55.

13. Kamolz LP, Lumena HB, Kazzinger M (2008) Tissue engineering for cutaneous wounds: an overview of current standards and possibilities. Eur Surg; 40: 19-26.

14. Pasek J, Opara J, Pasek T, Szwejkowski W, Sieron A (2007) The meaning of Quality of Life in rehabilitation. Physiotherapy; 15: 3-8.

15. Borg MJ, Marcuccio F, Poerio AM, Vangone A (1996) Magnetic fields in physical therapy. Experience in orthopedics and traumatology rehabilitation. Minerva Med; 87: 495-497.

16. Taradaj J, Franek A, Dolibog P, Cierpka L, Błaszczak E (2008) The physical treatment of chronic wound healing. Practical Rehabilitation; I: 34-35. 